\title{
Evidências de validade da versão brasileira da Gritical Thinking Disposition Scale
}

Validity evidence of the Critical Thinking Disposition Scale, Brazilian version Evidencias de validez de la versión brasileña de la Critical Thinking Disposition Scale

Franciane Silva Luiz' ${ }^{1}$ id https://orcid.org/0000-0002-5509-6703

Isabel Cristina Gonçalves Leite ${ }^{1}$ io https://orcid.org/0000-0003-1258-7331

Pedro Henrique Berbert de Carvalho ${ }^{1}$ io https://orcid.org/0000-0002-4918-5080

Vilanice Alves de Araújo Püschel ${ }^{2}$ io https://orcid.org/0000-0001-6375-3876

Luciene Muniz Braga ${ }^{3}$ io https://orcid.org/0000-0002-2297-395x

Herica Silva Dutra ${ }^{1}$ io https://orcid.org/0000-0003-2338-3043

Nádia Fontoura Sanhudo ${ }^{1}$ io https://orcid.org/0000-0001-9714-2854

Fábio da Costa Carbogim ${ }^{1}$ io https://orcid.org/0000-0003-2065-5998

\section{Como citar:}

Luiz FS, Leite IC, Carvalho PH, Püschel VA,

Braga LM, Dutra HS, et al. Evidências de

validade da versão brasileira da Critical Thinking Disposition Scale. Acta Paul Enferm. 2021;34:AAPE00413.

DOI

http://dx.doi.org/10.37689/actaape/2021A000413

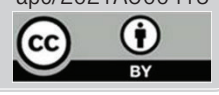

Descritores

Educação em enfermagem; Estudantes; Psicometria; Pensamento; Atitude do pessoal de saúde

Keywords

Education, nursing; Students; Psychometry; Thinking; Attitude of health personnel

\section{Descriptores}

Educación en enfermeira; Estudiantes; Psicometría; Pensamiento; Actitud del personal de salud

Submetido 1 de Março de 2020 Aceito 20 de Agosto de 2020

\section{Autor correspondente}

Fábio da Costa Carbogim Email: fabiocarbogim@gmail.com

\section{Resumo}

Objetivo: Realizar a tradução, adaptação cultural, análise de evidências de validade e confiabilidade da Critical Thinking Disposition Scale para o português do Brasil.

Métodos: Estudo metodológico que envolveu tradução inicial, síntese das traduções, retrotradução, avaliação por comitê de especialistas (evidências de validade de conteúdo), pré-teste e envio aos desenvolvedores para avaliação. Após essa etapa foi ainda realizada a avaliação de evidências de validade baseadas na estrutura interna do instrumento e de confiabilidade.

Resultados: A versão final da escala obteve coeficiente de validade de conteúdo com variação entre 0,88 e 0,96 para os itens. 0 tempo de aplicação variou entre 11 e 20 minutos. Análise fatorial exploratória indicou estrutura interna formada por um único fator com cargas fatoriais adequadas para todos os itens, bem como boa correlação item-total. 0 instrumento apresentou adequada evidência de confiabilidade (coeficiente Ômega) igual a 0,79.

Conclusão: 0 instrumento foi traduzido, adaptado, possui evidências de validade de conteúdo, de validade baseada na estrutura interna do instrumento e confiabilidade adequadas à cultura brasileira.

\section{Abstract}

Objective: To translate, culturally adapt, analyze the validity and reliability evidence of the Critical Thinking Disposition Scale for Brazilian Portuguese.

Methods: A methodological study that involved initial translation, synthesis of translations, back-translation, assessment by a committee of experts (content validity evidence), pre-test and sending to developers for assessment. After this stage, validity evidence based on the instrument's internal structure and reliability was also performed.

Results: The final version obtained a content validity coefficient with a variation between 0.88 and 0.96 for all items. Application time varied between 11 and 20 minutes. Exploratory factor analysis indicated an internal structure formed by a single factor with adequate factor loads for all items, as well as a good item-total correlation. The instrument presented adequate evidence of reliability (Omega coefficient) equal to 0.79 .

Conclusion: The instrument was translated, adapted, has content validity evidence based on the instrument's internal structure and reliability appropriate to the Brazilian culture. 


\section{Resumen}

Objetivo: Realizar la traducción, adaptación cultural, análisis de evidencias de validez y fiabilidad de la Critical Thinking Disposition Scale al portugués de Brasil.

Métodos: Estudio metodológico que incluyó traducción inicial, síntesis de las traducciones, retrotraducción, evaluación por comité de especialistas (evidencias de validez de contenido), prueba piloto y envío a los creadores para evaluación. Después de esta etapa, además fue realizada la evaluación de evidencias de validez basadas en la estructura interna del instrumento y de fiabilidad.

Resultados: La versión final de la escala obtuvo coeficiente de validez de contenido con variación entre 0,88 y 0,96 en los ítems. El tiempo de aplicación varió entre 11 y 20 minutos. El análisis factorial exploratorio indicó una estructura interna formada por un único factor con cargas factoriales adecuadas en todos los ítems, así como una buena correlación ítem-total. El instrumento presentó una evidencia de fiabilidad adecuada (coeficiente Omega) igual a 0,79.

Conclusión: El instrumento fue traducido, adaptado, posee evidencias de validez de contenido, de validez basada en la estructura interna del instrumento y fiabilidad adecuada para la cultura brasileña.

\section{Introdução}

Formar profissionais com capacidade de pensar criticamente e tomar decisóes complexas tem sido foco das diretrizes curriculares para os cursos de graduação da área de saúde. ${ }^{(1,2)}$ Nesse campo, o pensamento crítico (PC) é compreendido como a disposição em aplicar habilidades de reflexão intencionada, com base em evidências científicas, a partir de processos mentais superiores que reúnem pistas para a resolução de problemas. ${ }^{(2-4)}$

A literatura destaca que a atitude de disposição para o PC ocupa papel fundamental na aplicação efetiva da habilidade do pensamento crítico. ${ }^{(4,5)}$ Dessa forma, características como mente aberta, curiosidade, honestidade em enfrentar preconceitos pessoais, busca ativa da verdade, sistematicidade, perseverança são padróes mentais de disposição para o desenvolvimento do pensamento crítico. ${ }^{(2,6)}$ Esses padróes podem ser aprendidos por meio de atividades de orientação e operaçôes que agucem a metacognição (refletir sobre o próprio pensamento). ${ }^{(2)}$ Cabe destacar que o consenso internacional sobre $\mathrm{PC}$ o define como um processo de autorregulação para analisar e definir o que fazer em determinada situação. A disposição para o PC se relaciona com a motivaçáo interna em desempenhar determinada habilidade para se engajar na solução de problemas e tomada de decisão. ${ }^{(6)}$ Logo, as habilidades de PC só serão aplicadas se houver a disposição ou atitude de disposiçáo para tal. Ambas, disposição e habilidades, podem ser mobilizadas por atividades problematizadoras. ${ }^{(7)}$

Estudos $^{(7,8)}$ têm evidenciado a relação positiva entre metodologias de ensino que estimulam e mobilizam o estudante a desenvolver disposição de PC e a uma imersão crítica na realidade em que se in- serem, visando à resolução de problemas. Pesquisa ${ }^{(9)}$ realizada com 102 estudantes de medicina da Universidade de Pequim, acompanhados por seis meses, destacou melhora nos escores para disposição do PC com a utilização da aprendizagem baseada em problemas. Outro estudo avaliou comparativamente o efeito do ensino formal (grupo controle) e a aprendizagem baseada em casos (ABC; grupo experimental) sobre a disposição do $\mathrm{PC}$ em 80 estudantes de enfermagem por 18 semanas. ${ }^{(10)}$ Foi verificado que o grupo experimental, ensinado por meio do $\mathrm{ABC}$, apresentou escores estatisticamente significativos para disposição do $\mathrm{PC}$ em comparação ao grupo controle.

Tendo por foco a avaliação, existem poucos instrumentos não comerciais que mensurem de maneira válida e precisa e de forma rápida, a disposição do PC. ${ }^{(3,11)}$ Nesse sentido, Sosu ${ }^{(12)}$ criou a escala Critical Thinking Disposition Scale (CTDS), composta por 11 itens, distribuídos nas dimensôes abertura crítica e ceticismo reflexivo, que mensuram a disposição para o PC.

Assim, tendo em vista a relevância do instrumento para o ensino de enfermagem/saúde e a inexistência no Brasil de instrumento que permita mensurar a disposição para o PC, justifica-se a realização deste estudo, que tem como objetivo realizar a tradução, a adaptaçáo cultural, análise de evidências de validade e confiabilidade da CTDS para o português do Brasil.

\section{Métodos}

Estudo metodológico conduzido para adaptação transcultural da CTDS para o português do Brasil. ${ }^{(13,14)} \mathrm{O}$ 
processo de tradução, adaptação cultural para a língua portuguesa do Brasil e a utilização da CTDS foram autorizados pelo autor da escala.

A CTDS é um instrumento constituído por 11 itens que medem duas dimensóes relacionadas à disposição para o PC: a abertura crítica e o ceticismo reflexivo. A subescala abertura crítica, composta por sete itens (letras a, b, c, d, e, g, h), reflete a tendência de estar atento a novas ideias, realizar críticas na avaliaçáo dessas ideias e modificar o pensamento diante de evidências convincentes. Já a subescala ceticismo reflexivo, constituída por quatro itens (letras $f, i, j, k$ ), avalia a tendência de aprender com experiências passadas e de questionar evidências. Os itens são dispostos numa escala Likert de pontos e as respostas variam de 1 a 5, valores que correspondem, respectivamente, aos termos "Discordo Totalmente, Discordo, Não Concordo e Nem Discordo, Concordo e Concordo Totalmente".

A disposição para o PC pode ser classificada em baixa, moderada ou alta. Para realizar a classificação, devem-se somar os 11 itens, encontrando-se um escore que varia de 11 e 55 pontos. $^{(12)}$

$\mathrm{O}$ estudo foi desenvolvido em duas fases, sendo a primeira para tradução e adaptação cultural, conforme proposta de Beaton, Bombardier, Guillemin e Ferraz. ${ }^{(15)}$ A primeira fase foi constituída por seis etapas: tradução inicial, síntese das traduçóes, retrotradução, comitê de especialistas, pré-teste e envio de documentação aos desenvolvedores para avaliação do processo de adaptação. $\mathrm{Na}$ segunda fase, foi realizada a avaliação de evidências de validade baseadas na estrutura interna do instrumento e confiabilidade.

$\mathrm{Na}$ etapa de tradução inicial, o instrumento foi traduzido por dois profissionais, de forma independente, com domínio da língua inglesa e que possuíam o português como língua nativa. Ressalta-se que o tradutor 1, diferentemente do tradutor 2, ficou ciente dos conceitos a serem examinados e teve acesso ao artigo que versava sobre o instrumento a ser validado.

Na etapa de síntese, as duas traduçôes para a língua portuguesa do Brasil (T1 e T2) foram comparadas pelos dois tradutores e um dos pesquisadores, que, de forma sintética e consensual, produziram a versão síntese em português (T12).
Na etapa de retrotradução, a T12 foi traduzida de forma reversa para a língua original, por dois profissionais nativos da língua do instrumento original e com domínio da língua portuguesa, de forma cega e independente, dando origem às retrotraduçôes (RT1 e RT2).

Posteriormente, a versão original do instrumento em inglês, cada tradução do inglês para o português (T1 e T2), a versão síntese (T12) e as retrotraduçôes do português para o inglês (RT1 e RT2) foram submetidas a um comitê de seis especialistas, originando um consenso das traduçóes, que constituiu a versão utilizada no pré-teste.

No presente trabalho, o comitê foi composto por seis integrantes, dos quais cinco especialistas e um tradutor juramentado (com conhecimento em processo de tradução e adaptação cultural e em PC). O processo de avaliação por especialista teve por objetivo garantir a adaptação cultural do instrumento, com destaque para a equivalência semântica, idiomática e cultural do instrumento a ser utilizado no pré-teste. Então, no pré-teste, a versão que foi consensuada pelos especialistas foi aplicada em uma amostra de conveniência de 33 estudantes do curso de graduação em enfermagem de uma instituição pública de Minas Gerais. Nessa etapa, verificou-se o grau de clareza dos itens traduzidos e o tempo despendido para preenchimento do instrumento.

Para finalizar a primeira fase (tradução e adaptação cultural), salienta-se que o desenvolvedor da CTDS assegurou que todas as etapas propostas foram seguidas e que o instrumento atingiu uma tradução admissível.

$\mathrm{Na}$ segunda fase, procedeu-se ao processo de busca de evidências de validade interna e confiabilidade do instrumento traduzido e adaptado para o português do Brasil. Para tal, a coleta dos dados ocorreu com estudantes do curso de graduação da Faculdade de Enfermagem da Universidade Federal de Juiz de Fora (UFJF).

O cálculo amostral seguiu orientaçóes que sugerem utilizar uma proporcionalidade de 10 participantes para cada item do instrumento. ${ }^{(16,17)} \mathrm{A}$ $\mathrm{CTDS}^{(12)}$ possui uma estrutura fatorial composta por 11 itens, resultando em uma amostra mínima de 110 estudantes. Fizeram parte da amostra da 
investigação 179 estudantes que responderam ao instrumento.

Os critérios de inclusão foram: estudantes do Curso de Graduação em Enfermagem da UFJF, com idade maior ou igual a 18 anos e que se encontravam em aula durante a aplicação dos instrumentos. Foram excluídos os alunos afastados do curso por trancamento de matrícula ou motivo de saúde e os que participaram do pré-teste.

O banco de dados foi elaborado no Microsoft Office Excel 2010. Para as análises estatísticas foram utilizados os programas Statical Package for the Social Sciences (SPSS) versão 22.0 e o software JASP versão 0.11.1.

Para análise de evidências de validade de conteúdo e evidências de validade baseada na estrutura interna, foi aplicado o coeficiente de validade de conteúdo (CVC) e a análise fatorial exploratória, respectivamente. Para avaliar a distribuição dos dados, foram adotados como indicativo de distribuição não-normal dos dados: assimetria (maior do que 3), curtose (maior do que 7), e coeficiente de Mardia (maior do que 5). ${ }^{(16,17)}$ Em seguida, os dados foram analisados em busca da existência de outliers multivariados (distância quadrada de Mahalanobis - $D^{2}$ ). ${ }^{(16,17)}$

A análise fatorial exploratória foi realizada utilizando como método estimativo a Fatoração do Eixo Principal com rotação oblíqua (oblimin direto) e o critério de Kaiser (autovalor mínimo = 1) para extração fatorial. ${ }^{(18)} \mathrm{O}$ gráfico de sedimentação (scree-plot) foi inspecionado e a técnica de análise paralela foi utilizada. ${ }^{(16,17)}$ Para identificar se a matriz de dados era fatorável, foram utilizados o teste de KaiserMeyer-Olkin (KMO) e o teste de esfericidade de Bartlett. O KMO foi considerado adequado se superior a 0,80 e o teste de esfericidade de Bartlett $\mathrm{p}$ $<0,05$. $^{(16,17)}$

A matriz de carga fatorial foi analisada para identificar itens e sua correspondência com fatores. Um ponto de corte de 0,40 foi considerado adequado para a retenção de itens. ${ }^{(19)}$ Itens com carga fatorial $(\lambda)$ maior que 0,32 foram considerados cargas cruzadas. ${ }^{(19)}$ Por fim, foi analisada a correlação item-total. ${ }^{(16,17)}$

A estimativa de confiabilidade da estrutura interna foi obtida por meio do coeficiente Ômega
(95\%IC). ${ }^{(20)}$ Valores acima de 0,70 foram considerados adequados. ${ }^{(20)}$

Todas as etapas da investigação foram realizadas de acordo com a Resolução 466/2012 do Conselho Nacional de Saúde para pesquisas envolvendo seres humanos. O projeto foi aprovado por Comitê de Ética em Pesquisa (parecer 2.404.971, CAAE 77669417.0.0000.5147) e os participantes foram informados sobre o estudo e assinaram o Termo de Consentimento Livre e Esclarecido.

\section{Resultados}

As três primeiras etapas, referentes à tradução, síntese e retrotradução, foram realizadas de forma satisfatória, tendo sido necessária a realização de modificaçôes ínfimas. Todas as sugestôes realizadas foram amplamente discutidas até a obtenção da versão adaptada.

$\mathrm{Na}$ quarta etapa, que diz respeito à revisão por um comitê, participaram seis especialistas, cuja idade variou entre 30 e 53 anos, com média de 41,5 anos. Destes, quatro $(66,7 \%)$ eram do sexo feminino e autodeclarados da raça branca, metade (três) era residente no Estado de São Paulo e a outra metade no Estado de Minas Gerais. No que se refere à escolaridade, todos eram graduados em enfermagem, sendo três doutores, dois mestres e um possuía título de especialista. No que tange à experiência, metade (três) do comitê de especialistas possuía conhecimento sobre a temática de $\mathrm{PC}$, dois sobre o método de tradução e adaptação cultural e validação e um tinha conhecimento tanto da temática quanto do método.

Ainda nesta etapa, a síntese das duas traduçóes (T12) foi avaliada pelo Comitê de especialistas em termos dos quesitos de equivalência transcultural de cada item. Para tanto, eles consideraram todas as versôes (T1, T2, RT1 e RT2) e todos os relatórios provenientes delas. Nas etapas I, II, III e IV, quando houve discordância em relação ao termo ou expressão, optou-se pelo mais usual na língua portuguesa, assegurando-se em todos os itens a manutenção das equivalências semântica, idiomática, experiencial e conceitual. 
Após essa avaliação, os juízes julgaram necessário realizar alteraçôes no item "g" e no título. Por exemplo, o tradutor 1 traduziu a expressão "biggerpicture" para "como um todo" e o tradutor 2 "no contexto geral". Como se trata de uma figura de linguagem, junto com os dois tradutores, os especialistas decidiram adotar a expressão "no contexto geral". Em relação ao título, em sua versão original, "Critical Thinking Disposition Scale", a principal repercussão recaiu sobre o termo "disposition". Apesar de ambos os tradutores optarem pelo termo "disposição", inicialmente, compreendeu-se que o termo mais próximo era “atitude". Contudo, após realização da avaliação da retrotradução e avaliação pelo autor da escala, o termo "disposição" foi eleito, considerando que o termo "atitude" não é compatível idiomaticamente na língua original do instrumento.

$\mathrm{Na}$ etapa seguinte, denominada pré-teste, a versão pré-final (consensuada pelos especialistas) foi aplicada, de forma aleatória, e analisada por 33 estudantes em um teste piloto. A idade dos participantes variou entre 22 e 38 anos, com média de 27,9 anos. A maioria $(81,8 \%)$ era do sexo feminino e autodeclarada da raça branca $(60,6 \%)$. O tempo de aplicação do instrumento variou entre 11 e 20 minutos. Optou-se pela aplicação individual ou autoadministrável do instrumento, apesar de poder ser aplicado coletivamente.

No que se refere ao grau de clareza dos itens traduzidos, 90\% ( $\mathrm{n}=30)$ dos estudantes consideraram o título claro ou bastante claro, $87 \%(\mathrm{n}=29)$ julgaram o enunciado claro ou bastante claro e $84,5 \%$ $(n=28)$ avaliaram os itens da escala do tipo Likert como claros ou bastante claros. Contudo, um estudante $(3 \%)$ considerou como "pouco claro" o item " $h$ ". Optou-se por não o modificar, pois a dúvida estava relacionada à expressão "convicçóes mais fortes”. Trata-se de uma afirmação importante para a subescala abertura crítica, não tendo sido encontrada outra expressão adequada para substituição.

Dessa forma, após ajustes das consideraçóes realizadas pelos estudantes, os autores optaram por retornar à etapa IV e solicitar aos juízes que reavaliassem o grau de clareza do instrumento. Dessa vez, o grau de concordância entre juízes variou de 0,88 a
0,96 para os itens e o CVC geral da escala resultou em 0,91 , como pode ser observado na tabela 1 .

Tabela 1. Evidências de validade de conteúdo por item

\begin{tabular}{|c|c|}
\hline Item & CVC item \\
\hline a. Eu frequentemente estou à procura de novas ideias & 0,88 \\
\hline $\begin{array}{l}\text { b. Eu frequentemente uso novas ideias para definir (ou modificar) a maneira } \\
\text { como faço as coisas }\end{array}$ & 0,92 \\
\hline c. Eu utilizo mais do que uma fonte para encontrar informações & 0,96 \\
\hline d. É importante justificar as escolhas que eu faço & 0,92 \\
\hline $\begin{array}{l}\text { e. É importante compreender o ponto de vista de outras pessoas com relação } \\
\text { a um determinado assunto }\end{array}$ & 0,92 \\
\hline $\begin{array}{l}\text { f. Eu normalmente penso sobre as várias consequências de uma decisão } \\
\text { antes de agir }\end{array}$ & 0,92 \\
\hline g. Eu, durante uma discussão, normalmente tento pensar no contexto geral & 0,88 \\
\hline $\begin{array}{l}\text { h. Às vezes, eu encontro um bom argumento que contesta algumas das } \\
\text { minhas convicções mais fortes }\end{array}$ & 0,88 \\
\hline $\begin{array}{l}\text { i. Eu normalmente verifico a credibilidade das fontes de informação antes de } \\
\text { fazer julgamentos }\end{array}$ & 0,96 \\
\hline $\begin{array}{l}\text { j. Eu frequentemente reavalio minhas experiências para que eu possa } \\
\text { aprender com elas }\end{array}$ & 0,88 \\
\hline $\begin{array}{l}\text { k. Eu frequentemente penso sobre minhas ações para ver se eu posso } \\
\text { melhorá-las }\end{array}$ & 0,88 \\
\hline CVC global & 0,91 \\
\hline
\end{tabular}

CVC - coeficiente de validade de conteúdo

$\mathrm{Na}$ segunda fase do processo de validação, procedeu-se à busca de evidências de validade baseada na estrutura interna e confiabilidade do instrumento traduzido e adaptado para o português do Brasil.

Dos 179 estudantes de graduação em enfermagem que responderam ao teste, $50,3 \%$ estavam cursando o ciclo básico e 49,7\%, o ciclo profissionalizante; com média de idade de 22,3 anos (DP: 5,7). A maioria $(84,9 \%)$ dos participantes era do sexo feminino, $57 \%$ autodeclarados da raça branca e $74,3 \%$ tinham renda familiar de até cinco salários mínimos.

A análise de distribuição dos dados não indicou valores elevados de assimetria e curtose. De igual modo, o coeficiente de Mardia foi inferior a 5. Em conjunto, esses indicam a normalidade dos dados. Não foi identificada presença de outliers multivariados.

$\mathrm{O}$ teste de esfericidade de Bartlett foi significativo $\left(\chi^{2}(55)=416,671, p<0,0001\right)$, e a medida $\mathrm{KMO}$ foi de 0,81 . Solução fatorial indicou a presença de dois fatores que juntos explicam 44,88\% da variância total dos dados (Tabela 2). Os autovalores observados para os fatores 1 e 2 são adequados, correspondendo a 3,585 e 1,352, respectivamente. A estrutura interna identificada no presente estudo 
difere daquela originalmente proposta por Sosu. ${ }^{(12)}$ A solução fatorial sugerida é de difícil interpretação, já que itens que originalmente formavam o fator "abertura crítica" carregaram no fator "ceticismo reflexivo", e vice-versa. Em adição, foi observada carga fatorial limítrofe para dois itens (letras "d" e "h"), bem como presença de carga cruzada para os fatores 1 e 2 para dois itens (letras " $h$ " e "i"). Isso reforça o fato de que a estrutura de dois fatores identificada não é adequada e sugere que outra estrutura interna poderia ser mais ajustada para avaliação da disposição para o PC. De fato, análise paralela contesta a existência de dois fatores (Figura 1). O autovalor obtido para o fator 2 pelo critério da análise paralela é superior ao obtido pela análise fatorial dos dados.

Tabela 2. Autovalores, variância explicada por fator, correlação item-total, e extração de fatores da CTDS

\begin{tabular}{l|cccccc|}
\hline Itens & Autovalores & $\begin{array}{c}\text { Variância } \\
\text { explicada } \\
\text { por fator } \\
(\%)\end{array}$ & $\begin{array}{c}\text { Correlação } \\
\text { item-total }\end{array}$ & \multicolumn{2}{c}{$\begin{array}{c}\text { Solução fatorial } \\
\text { inicial }(\lambda)\end{array}$} & $\begin{array}{c}\text { Solução } \\
\text { fatorial } \\
\text { final }(\lambda)\end{array}$ \\
\hline a & 3,585 & 32,59 & 0,39 & 0,01 & 0,79 & 0,51 \\
b & 1,352 & 12,29 & 0,47 & 0,23 & 0,65 & 0,59 \\
\hline c & 0,987 & 8,98 & 0,40 & 0,08 & 0,71 & 0,52 \\
d & 0,903 & 8,21 & 0,38 & 0,40 & 0,28 & 0,49 \\
\hline e & 0,846 & 7,70 & 0,39 & 0,56 & 0,12 & 0,50 \\
f & 0,768 & 6,98 & 0,43 & 0,76 & $-0,04$ & 0,56 \\
\hline$g$ & 0,669 & 6,08 & 0,52 & 0,72 & 0,14 & 0,64 \\
h & 0,552 & 5,02 & 0,39 & 0,33 & 0,40 & 0,50 \\
i & 0,490 & 4,45 & 0,50 & 0,33 & 0,59 & 0,63 \\
j & 0,460 & 4,18 & 0,51 & 0,64 & 0,24 & 0,65 \\
\hline k & 0,388 & 3,52 & 0,52 & 0,63 & 0,28 & 0,66 \\
\hline
\end{tabular}

CTDS - Critical Thinking Disposition Scale; \% - percentual de variância explicada por fator; $\lambda$ - carga fatorial; Método de extração pela Fatoração do Eixo Principal (rotação oblíqua, oblimin direto); Cargas fatoriais em negrito representam a pertinência do item em seu fator

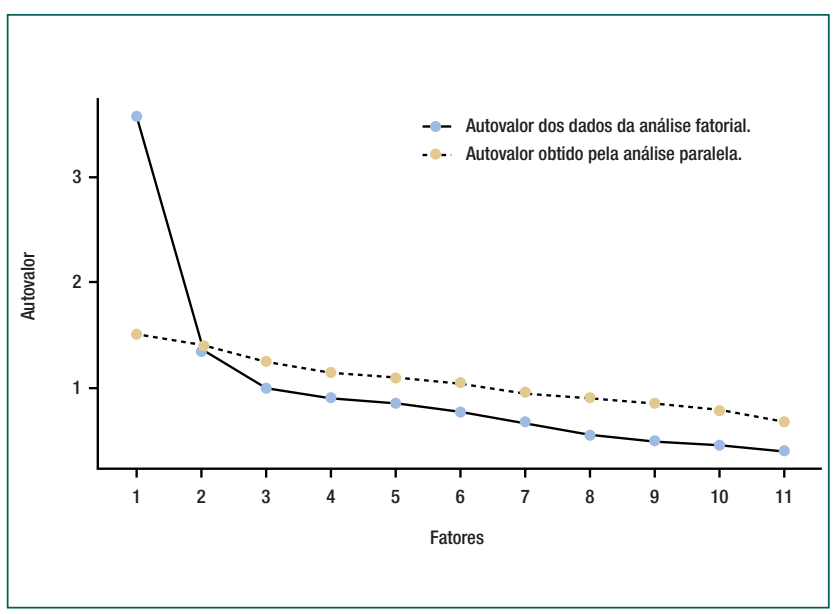

Figura 1. Gráfico de sedimentação indicativo dos números de fatores obtidos pela análise fatorial exploratória (autovalores superiores a 1) e pela análise paralela
Deste modo, análise fatorial exploratória foi novamente conduzida fixando a solução em um único fator (Tabela 2). A carga fatorial de todos os itens foi superior ao ponto de corte de 0,40. A menor carga fatorial identificada $(\lambda=0,49)$ foi para o item " $\mathrm{d}$ ". A correlação item-total foi adequada para todos os itens. Por fim, o instrumento apresentou coeficiente Ômega para a escala global igual a 0,79 (IC95\% = $0,74-0,83)$. Tais indicadores sugerem que uma estrutura de único fator formada por 11 itens é capaz de representar a disposição para o PC.

\section{Discussão}

Estudos $^{(3,11)}$ sobre o PC vêm se ampliando no Brasil, contudo ainda são poucos os que se baseiam em ferramentas capazes de avaliar/medir essa competência de maneira válida e confiável. Internacionalmente, apenas o Cornell Critical Thinking Test (CCTT), a California Critical Thinking Disposition Inventory (CCTDI) e a Critical Thinking Disposition Scale (CTDS) mensuram a disposição para o PC, mas nenhum desses instrumentos teve suas evidências de validade avaliadas para o português do Brasil. (3,7,11)

A CTDS ${ }^{(12)}$ é um instrumento que possui evidências de validade e confiabilidade, assim como o CCTT e CCTDI, porém se destaca por ser um instrumento objetivo, de fácil aplicação e não comercial. Ademais, a partir dos padrôes de confiabilidade e validade, o processo de tradução e adaptação cultural mostrou-se satisfatório. ${ }^{(13-15)}$

A retrotradução para o inglês, realizada por dois tradutores de forma independente, apontou que a versão traduzida e adaptada para o contexto brasileiro manteve correspondência com a versão do instrumento original. O resultado final de tradução e adaptação da CTDS possibilitou adequação de termos traduzidos, respeitando-se todas as equivalências e grau de concordância nos itens maior que $80 \%{ }^{(14)}$

Não se identificou na literatura, até o momento, estudos que tenham realizado tradução e adaptação cultural da CTDS de forma a viabilizar a comparação nesse quesito. Destaca-se que o rigor no processo de tradução e validação de um instrumento 
de medida tem sido fortemente recomendado, pois produz evidências de validade em outro contexto cultural. ${ }^{(13-15)}$

Foram obtidas evidências de validade com base na estrutura interna do instrumento. Em uma primeira exploração dos dados uma estrutura formada por dois fatores foi obtida. Contudo, análise de interpretação dos itens e fatores, bem como a paralela indicaram inadequação dessa estrutura. ${ }^{(16,17)} \mathrm{A}$ análise fatorial exploratória foi reconduzida e indicou que a versão brasileira da CTDS é mais bem ajustada com um único fator que representa a disposição para o PC. Os 11 itens originais da escala apresentaram carga fatorial satisfatória para sua retenção, bem como correlação item-total adequada. Destaca-se que essa estrutura interna difere daquela obtida no estudo original de validação da CTDS, ${ }^{(12)}$ que indica a necessidade de estudos confirmatórios dessa estrutura para o contexto brasileiro. Dito de outra maneira é necessário avaliar se a disposição para o PC é mais bem representada por dimensóes como a "abertura crítica" e o "ceticismo reflexivo", como proposto por Sosu ${ }^{(12)}$ na validação da CTDS, ou se o construto pode ser descrito de modo unidimensional. No presente estudo, foi avaliada tanto uma estrutura de dois fatores, quanto uma estrutura unidimensional da CTDS, sendo essa última a de melhor ajuste. Estudos futuros devem avaliar modelos concorrentes de modo a contribuir com a fundamentação teórica sobre a disposição para o PC, bem como para confirmar os dados aqui indicados em outras realidades culturais.

No que se refere às análises de evidências de confiabilidade da CTDS, o instrumento apresentou coeficiente Ômega superior a 0,70 ( $\dot{\omega}=$ $0,79$; IC95\% $=0,74-0,83)$. Na versão original da CTDS, os resultados de consistência interna foram obtidos por meio do coeficiente alfa de Cronbach, sendo obtido valor de $0,79 .{ }^{(12)}$ Pesquisa realizada na Turquia, com uma amostra de 212 estudantes universitários, encontrou coeficiente alfa de Cronbach

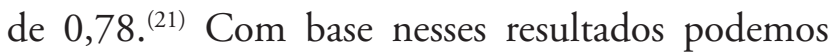
observar que nossos valores são similares àqueles obtidos para a escala global em estudos prévios de validação da CTDS. ${ }^{(12,21)}$ Destacamos, que dada a natureza da escala Likert de resposta a CTDS, utili- zamos o coeficiente Ômega, mais indicado para esse tipo de análise. ${ }^{(20)}$

Assim, por meio das análises de busca de evidências de validade baseadas na estrutura interna do instrumento e de confiabilidade, assegura-se que a versão adaptada da CTDS brasileira pode ser aplicada em atividades em que se queira avaliar disposição do pensamento. Trata-se de uma ferramenta importante para a pesquisa com acadêmicos e profissionais, incluindo a área de enfermagem/saúde.

Como limitaçáo do estudo destaca-se o reduzido número de participantes. Apesar de seguir recomendaçóes sobre o número mínimo de participantes por itens do instrumento, considera-se que amostras acima de 300 indivíduos são mais adequadas para avaliar a confiabilidade e a qualidade da análise fatorial. ${ }^{(16,17)}$ Além disso, a amostra é composta por estudantes de um contexto específico, o que sabidamente pode afetar o ajuste do modelo. ${ }^{(16)}$

\section{Conclusão}

A tradução e a adaptação cultural da Critical Thinking Disposition Scale permitiram a adequação do instrumento ao contexto brasileiro. A Escala de Disposição do Pensamento Crítico apresentou evidências de validade de conteúdo, bem como evidências de validade baseadas na estrutura interna do instrumento e adequada estimativa de confiabilidade. $\mathrm{O}$ instrumento, adaptado à cultura brasileira, poderá ser útil para avaliar atividades acadêmicas e profissionais acerca da disposiçáo do pensamento crítico.

\section{Agradecimentos}

Agradecemos à Universidade Federal de Juiz de Fora pelo apoio financeiro a esta pesquisa.

\section{Colaborações}

Luiz FS, Leite ICG, Carvalho PHB, Püschel VAA, Braga LM, Dutra HS, Sanhudo NF e Carbogim FC 
contribuíram com a concepção do estudo, análise e interpretação dos dados, redação do artigo, revisão crítica relevante do conteúdo intelectual e aprovação da versão final a ser publicada.

\section{Referências}

1. Jiménez-Gómez MA, Cárdenas-Becerril L, Velásquez-Oyola MB, Carrillo-Pineda M, Barón-Díaz LY. Reflective and critical thinking in nursing curriculum. Rev Lat Am Enfermagem. 2019;27:e3173.

2. Carbogim FC, Oliveira LB, Püschel VA. Critical thinking: concept analysis from the perspective of Rodger's evolutionary method of concept analysis. Rev Lat Am Enfermagem. 2016 Sep;24:e2785.

3. Riegel F, Crossetti MD. Referenciais teóricos e instrumentos para avaliação do pensamento crítico na enfermagem e na educação. Rev Gaúcha Enferm. 2018;39(0):e20170097.

4. Cui C, Li Y, Geng D, Zhang H, Jin C. The effectiveness of evidencebased nursing on development of nursing students' critical thinking: $\mathrm{A}$ meta-analysis. Nurse Educ Today. 2018;65:46-53.

5. Carbogim FD, Barbosa AC, de Oliviera LB, de Sá Diaz FB, Toledo LV, Alves $\mathrm{KR}$, et al. Educational intervention to improve critical thinking for undergraduate nursing students: A randomized clinical trial. Nurse Educ Pract. 2018;33:121-6.

6. Facione PA. Critical thinking: a statement of expert consensus for purposes of educational assessment and instruction: Research findings and recommendations. Fullerton: California State University; 1990.

7. Oliveira LB, Díaz LJ, Carbogim FC, Rodrigues AR, Püschel VA. Effectiveness of teaching strategies on the development of critical thinking in undergraduate nursing students: a meta-analysis. Rev Esc Enferm USP. 2016;50(2):355-64.

8. López M, Jiménez JM, Martín-Gil B, Fernández-Castro M, Cao MJ, Frutos $M$, et al. The impact of an educational intervention on nursing students' critical thinking skills: A quasi-experimental study. Nurse Educ Today. 2020;85:104305.
9. Pu D, Ni J, Song D, Zhang W, Wang Y, Wu L, et al. Influence of critical thinking disposition on the learning efficiency of problembased learning in undergraduate medical students. BMC Med Educ. 2019;19(1):1.

10. Li S, Ye X, Chen W. Practice and effectiveness of "nursing casebased learning" course on nursing student's critical thinking ability: A comparative study. Nurse Educ Pract. 2019;36:91-6.

11. Carvalho EC, Oliveira-Kumakura AR, Morais SC. Clinical reasoning in nursing: teaching strategies and assessment tools. Rev Bras Enferm. 2017;70(3):662-8.

12. Sosu EM. The development and psychometric validation of a Critical Thinking Disposition Scale. Think Skills Creativity. 2013;9:107-19.

13. Fortes CP, Araújo AP. Check list para tradução e Adaptação Transcultural de questionários em saúde. Cad Saude Colet. 2019;27(2):202-9.

14. Souza AC, Alexandre NM, Guirardello EB. Propriedades psicométricas na avaliação de instrumentos: avaliação da confiabilidade e da validade. Epidemiol Serv Saude. 2017;26(3):649-59.

15. Beaton DE, Bombardier C, Guillemin F, Ferraz MB. Guidelines for the process of cross-cultural adaptation of self-report measures. Spine. 2000;25(24):3186-91.

16. Hair JF, Black WC, Babin BJ, Anderson RE, Tatham RL. Multivariate data analysis] (6 $6^{\mathrm{a}}$ ed.). New York, NY: Cengage Learning; 2009.

17. Kline P. An easy guide to factor analysis. New York (NY): Routledge; 2014.

18. Fabrigar LR, Wegener DT, MacCallum RC, Strahan EJ. Evaluating the use of exploratory factor analysis in psychological research. Psychol Methods. 1999;4(3):272-99.

19. Worthington R, Whittaker T. Scale development research: A content analysis and recommendations for best practice. Couns Psychol. 2006;34(6):806-38.

20. Reise SP, Bonifay WE, Haviland MG. Scoring and modeling psychological measures in the presence of multidimensionality. J Pers Assess. 2013;95(2):129-40.

21. Akin A, Hamedoglu MA, Arslan S, Akin Ü, Çelik E, Arslan ÇK. The Adaptation and Validation of the Turkish Version of the Critical Thinking Disposition Scale (CTDS). Int J Educ Res. 2015;6(1):31-5. 